\title{
Analytic semi-classical quantization of a QCD string with light quarks
}

\author{
T. J. Allen ${ }^{11}$, C. Goebel ${ }^{\dagger}$, M. G. Olsson ${ }^{\dagger}$ and S. Veseli \\ Dept. of Physics, Hobart \& William Smith Colleges, Geneva, New York 14456 \\ ${ }^{\dagger}$ Dept. of Physics, University of Wisconsin, 1150 University Avenue, Madison, Wisconsin 53706 \\ Fermi National Accelerator Laboratory, P.O. Box 500, Batavia, Illinois 60510
}

\begin{abstract}
We perform an analytic semi-classical quantization of the straight QCD string with one end fixed and a massless quark on the other, in the limits of orbital and radial dominant motion. Our results well approximate those of the exact numerical semi-classical quantization as well as our exact numerical canonical quantization.
\end{abstract}

\section{INTRODUCTION}

Linearly rising Regge trajectories are a prediction of both string and scalar confinement with one (or two) light quark(s). In a QCD string theory with one (or two) light spinless quark(s), the relation of the energy of the light degrees of freedom, $E$, to the the angular momentum and radial quantum numbers, $J$ and $n$, is well approximated by

$$
\frac{E^{2}}{(2) \pi a}, J+2 n+\frac{3}{2}
$$

where $a$ is the tension (linear energy density) of the string. The same relation holds exactly in scalar confinement, though with a denominator of $2 a$ instead of $\pi a$. We derive Eq. (1) analytically in semi-classical quantization of a straight string fixed at one end and with a massless and spinless quark at the other, in the limit of large radial quantum numbers $n$. The straight string approximation is an excellent approximation to the motion of a Nambu-Goto string; string curvature affects the energy and the angular momentum of the system very little [1]. The semi-classical result will follow from the evaluation of a single integral when one or both quarks are massless.

\section{DYNAMICS \& QUANTIZATION}

The energy and angular momentum of the system are its only conserved quantities. We would like to relate the energy to the angular momentum but the relationship of the angular momentum to the quark's transverse velocity (or to its angular velocity) is

\footnotetext{
${ }^{1}$ talk presented by T. J. Allen
} 


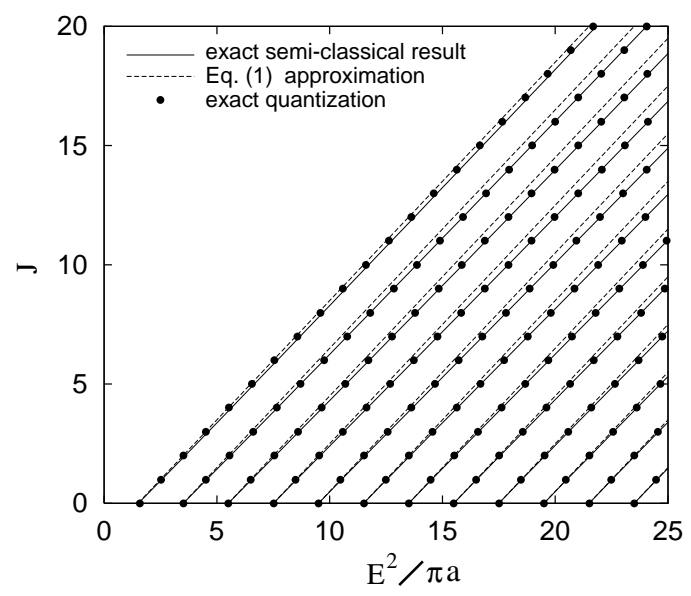

(a)

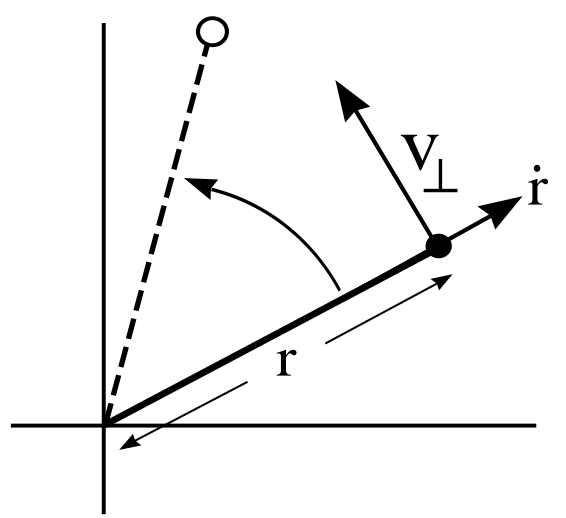

(b)

FIGURE 1. (a) Regge plot of angular momentum versus squared energy over $\pi a$. The numerical semiclassical quantized results agree nearly exactly with the exact numerical canonical results. Eq. (1) also agrees for $n \quad J$. (b) The configuration of a straight string with a quark on the end is described by the quark's position $\mathbf{r}$, its radial velocity $\dot{r}$ and its transverse velocity $v_{\text {? }}$.

transcendental and the transverse velocity cannot be eliminated analytically. Despite this difficulty, we can carry out semi-classical quantization analytically in the limit of large quantum numbers. This is not an essential limitation as semi-classical quantization is only valid in this limit.

The energy and angular momentum of the quark-string system, shown in Fig. 1(b), is well-known and given by [2]

$$
\begin{aligned}
& E=W_{r} \gamma_{?}+\operatorname{ar} \frac{\arcsin \left(v_{?}\right)}{v_{?}} ; \\
& J=W_{r} \gamma_{?} v_{?} r+a r^{2} @ \frac{\arcsin \left(v_{?}\right)}{2 v_{?}^{2}} \quad \frac{q \frac{v_{?}^{2}}{1} \mathrm{~A} ;}{2 v_{?}} \text {; }
\end{aligned}
$$

where

$$
\begin{array}{ll}
W_{r} & \mathrm{q} \frac{m_{r}^{2}+m^{2}}{p^{2}}=\mathrm{q} \frac{}{1 v_{?}^{2}} \mathrm{p} \overline{p^{2}+m^{2}} ; \\
\gamma_{?} & \left(1 \quad \frac{2}{p^{2}}\right)^{1=2}:
\end{array}
$$

\section{Dimensionless Variables}

To simplify the system, it pays to work in dimensionless units. We choose our units to be those of a system in uniform circular motion. The orbital energy and radius are $E_{0}=\overline{J \pi a}$ and $r_{0}=2 \overline{J=(\pi a)}$ respectively. We take the dimensionless energy, radius, 


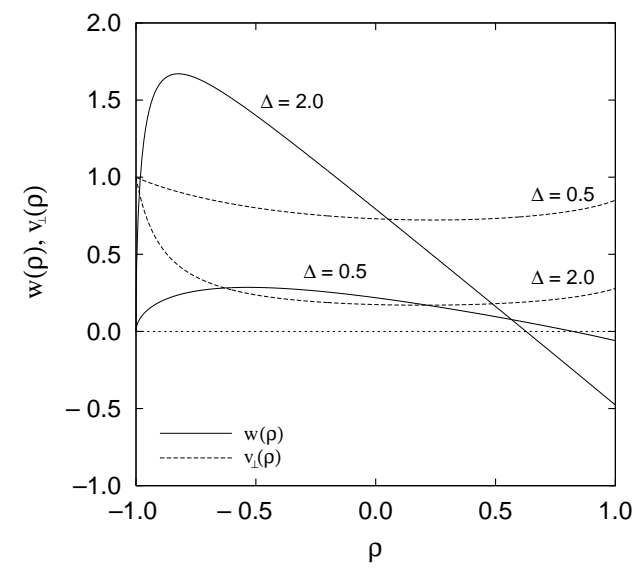

(a)

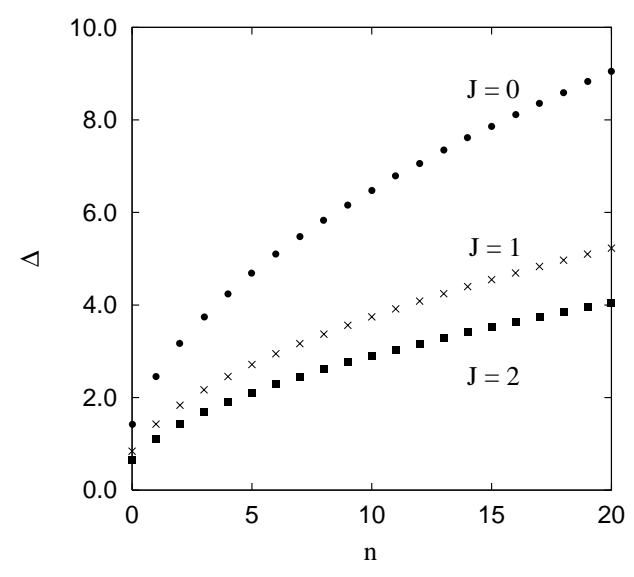

(b)

FIGURE 2. (a) The behavior of $w(\rho)$ and $v_{\text {? }}(\rho)$ for $\Delta=2$ and $\Delta=0$ 5. (b) $\Delta$ versus $n$ for $J=0 ; 1 ; 2$.

and quark radial energy to be given in terms of dimensionless numbers $\Delta, \rho$, and $w$ by

$$
\begin{array}{rl}
E=E_{0} & \mathrm{p} \overline{1+\Delta^{2}} ; \\
r=r_{0} & \mathrm{p} \frac{1+\Delta^{2}}{1+\rho \Delta ;} ; \\
W_{r}=E_{0} & w:
\end{array}
$$

When $\Delta=0$ the system is in uniform circular motion and lies along the leading Regge trajectory. Radial excitation corresponds to $\Delta>0$.

We can find $v_{\text {? }}$ and $w$ as functions of $\rho$ and $\Delta$ from Eqs. (2) and (3), made suitably dimensionless,

$$
\begin{aligned}
& w=\mathrm{q} \underset{1 v_{?}^{2}}{\mathrm{p}} \overline{1+\Delta^{2}} \frac{2}{\pi} S\left(v_{?}\right) \mathrm{p} \overline{1+\Delta^{2}}+\rho \Delta \quad ; \\
& 1+\frac{4}{\pi}\left(v_{?} S\left(v_{?}\right) \quad f\left(v_{?}\right)\right)^{\mathrm{hp}} \frac{\Delta^{2}}{1+\rho \Delta^{i_{2}}} \text {; } \\
& =2 v_{?}{ }^{h \mathrm{p}} \overline{1+\Delta^{2}}+\rho \Delta^{\mathrm{i} \mathrm{p}} \overline{1+\Delta^{2}} \text {; }
\end{aligned}
$$

where $S\left(v_{?}\right)=\frac{\arcsin \left(v_{?}\right)}{v_{?}}$ and $f\left(v_{?}\right)=\frac{1}{2 v_{?}} S\left(v_{?}\right) \quad$ q $\overline{1} \quad$. Figure 2(a) shows the dependence of $w$ and $v_{\text {? }}$ on $\rho$ for $\Delta=05$ and $\Delta=2: 0$.

\section{Turning Points}

In order to carry out semi-classical quantization, one must know the locations of the turning points of the classical motion. The turning points are the radii at which the radial momentum vanishes, $p_{r}=0$. For a massless quark, this implies that $w=0$, but because 
the quark is massless, the radial velocity need not vanish. The inner turning point occurs at $\rho=1$ and the outer turning point occurs in the interval

$$
\frac{\pi}{2} \quad 1 \quad \rho \quad 1:
$$

The lower limit is reached for large $\Delta$ and the upper limit for $\Delta=0$. The turning points for $\Delta=0: 5$ and $\Delta=2: 0$ can be seen in Fig. 2(a).

\section{Semi-Classical Quantization}

The Bohr-Sommerfeld quantization condition with the Langer correction [3] is

$$
{ }_{r}^{\mathrm{Z}_{+}}{ }_{r_{r}} d r=\pi n+\frac{1}{2} \quad ; \quad n=0 ; 1 ; 2 ;::: ;
$$

where the radial momentum is a function of radius and shifted angular momentum, $p_{r}=p_{r}\left(r ; J+\frac{1}{2}\right)$. In the case of a massless quark, we define the integral

$$
I(\Delta)=\frac{4}{\pi}_{1}^{\mathrm{Z}}{\stackrel{\rho}{\rho_{+}(\Delta)}}^{d} d \rho w(\rho ; \Delta) ;
$$

which is proportional to the integral in Eq. (12). The spectrum is computed from the relation

$$
\Delta I(\Delta)=\frac{2 n+1}{J+\frac{1}{2}} ;
$$

once $\Delta$ is expressed in terms of the energy through Eq. (6).

\section{Radial Dominant Motion}

Semi-classical quantization should become exact in the limit of $n \quad J$. For small $J$, large $n$ implies large $\Delta$, as can be seen from Fig. 2(b), which shows the dependence of $\Delta$ on $n$ for small values of $J$. This is the radial dominant regime.

Large $\Delta$ implies either $\rho=1$, or $v$ ? ! 0 and

$$
\left.w(\rho ; \Delta) \quad ! \quad \Delta 1 \frac{2}{\pi}(1+\rho) \quad\right) \quad \rho_{+}=\frac{\pi}{2} \quad 1:
$$

In the limit of large $\Delta$, the integral $I(\Delta)$ in Eq. (13) becomes simply $\Delta$.

$$
I(\Delta)^{\prime}{ }_{1}^{\mathrm{Z}}{ }_{\frac{\pi}{2}} 1 \text { d } w(\rho ; \Delta)=\Delta:
$$


The quantization condition (14) becomes

$$
\Delta I(\Delta)=\Delta^{2}=\frac{E^{2}}{\left(J+\frac{1}{2}\right) \pi a} \quad 1=\frac{2 n+1}{J+\frac{1}{2}} ;
$$

which is the same as Eq. (1).

\section{Angular Dominant Motion}

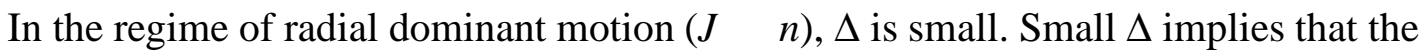
upper turning point is $\rho_{+}{ }^{\prime}$ 1. In this approximation we can also find an analytical form for $I(\Delta)$;

$$
\begin{aligned}
I(\Delta) & =\frac{4}{\pi}_{1}^{\mathrm{Z}+1} d \rho w(\rho ; \Delta)=\frac{8}{21} \quad \frac{3}{\pi} \quad \frac{\Gamma\left(\frac{2}{3}\right)}{\Gamma\left(\frac{7}{6}\right)} \Delta^{4=3} \\
& , \quad 13367 \Delta^{4=3}:
\end{aligned}
$$

Numerical evaluation of $I(\Delta)$ shows that Eq. (18) is a good approximation for $\Delta \quad 0: 4$. For larger $\Delta$ the radial dominant result of $\Delta$ is a better approximation.

\section{CONCLUSIONS}

We have performed a semi-classical quantization of the straight QCD string with massless and spinless quarks on the end and found an approximate spectrum that is similar in form to that of scalar confinement, though with a different Regge slope. In performing the quantization it is useful to use dimensionless variables related in an algebraic way to the energy and angular momentum.

In the case of massless quarks, a single integral function $I(\Delta)$ determines the spectrum. In the regime of radial dominant motion, this integral can be easily evaluated, and it is simply $\Delta$. This leads to the simple relation (1) that agrees well in the radial dominant regime with a full numerical canonical quantization of the system as well as to the exact numerical semi-classical quantization, as can be seen in Fig. 1(a).

\section{REFERENCES}

1. T.J. Allen, M.G. Olsson, and S. Veseli, Phys. Rev. D 59094011 (1999)

2. D. LaCourse and M. G. Olsson, Phys. Rev. D 39, 2751 (1989); C. Olson, M. G. Olsson, and K. Williams, Phys. Rev. D 45, 4307 (1992); M. G. Olsson and K. Williams, Phys. Rev. D 48, 417 (1993); M. G. Olsson and S. Veseli, Phys. Rev. D 51, 3578 (1995); M. G. Olsson, S. Veseli, and K. Williams, Phys. Rev. D 53, 4006 (1996).

3. R.E. Langer, Phys. Rev. 51, 669 (1937); M.S. Child, Molecular Collision Theory (Academic, New York, 1974). 